\title{
Ectopic expression of OsMADS45 activates the upstream genes Hd3a and RFT1 at an early development stage causing early flowering in rice
}

\author{
Jiun-Da Wang ${ }^{1}$, Shuen-Fang Lo ${ }^{2,4}$, Yan-Suan Li ${ }^{2}$, Po-Ju Chen ${ }^{2}$, Shih-Yun Lin ${ }^{1,5}$, Teh-Yuan Ho ${ }^{2}$, Jenq-Horng Lin ${ }^{1 *}$ \\ and Liang-Jwu Chen ${ }^{2,3^{*}}$
}

\begin{abstract}
Background: The rice gene, OsMADS45, which belongs to the MADS-box E class gene, participates in the regulation of floral development. Previous studies have revealed that ectopic expression of OsMADS45 induces early flowering and influences reduced plant height under short-day (SD) conditions. However, the regulation mechanism of OsMADS45 overexpression remains unknown. We introduce an OsMADS45 overexpression construct Ubi:OsMADS45 into TNG67 plants (an Hd1 (Heading date 1) and Ehd1 (Early heading date 1) defective rice cultivar grown in Taiwan), and we analyzed the expression patterns of various floral regulators to understand the regulation pathways affected by OsMADS45 expression.
\end{abstract}

Results: The transgenic rice exhibit a heading date approximately 40 days earlier than that observed in TNG67 plants, and transgenic rice display small plant size and low grain yield. OsMADS45 overexpression did not alter the oscillating rhythm of the examined floral regulatory genes but advanced (by approximately 20 days) the up-regulate of two florigens, Hd3a (Heading Date 3a) and RFT1 (RICE FLOWERING LOCUS T1) and suppressed the expression of Hd1 at the juvenile stage. The expression levels of OSMADS14 and OsMADS18, which are two well-known reproductive phase transition markers, were also increased at early developmental stages and are believed to be the major regulators responsible for early flowering in OsMADS45-overexpressing transgenic rice. OsMADS45 overexpression did not influence other floral regulator genes upstream of Hd1 and Ehd1, such as OsGl (OsGIGANTEA), Ehd2/Os/d1/RID1 and OSMADS50.

Conclusion: These results indicate that in transgenic rice, OsMADS45 overexpressing ectopically activates the upstream genes Hd3a and RFT1 at early development stage and up-regulates the expression of OsMADS14 and OsMADS18, which induces early flowering.

Keywords: Floral regulatory genes; Hd1; Hd3a; OsMADS45; RFT1; Rice

\section{Background}

The plant developmental transition from vegetative to reproductive growth is regulated by multiple genes and environmental factors, such as temperature and photoperiod (Koornneef et al. 1998; Wilczek et al. 2010). In rice, this transition influences the time of floral heading.

\footnotetext{
* Correspondence: jhlin@dragon.nchu.edu.tw; ljchen@nchu.edu.tw 'Department of Life Sciences, National Chung Hsing University, Taichung 402, Taiwan

${ }^{2}$ Institute of Molecular Biology, National Chung Hsing University, Taichung 402, Taiwan

Full list of author information is available at the end of the article
}

The heading date is an important agronomic trait of cultivated rice for adaption to variable growth season and photoperiod changes and is also associated with biomass, plant size and grain productivity (Xue et al. 2008; Endo-Higashi and Izawa 2011). Rice are cultivated within a wide range of latitudes ranging from $55^{\circ} \mathrm{N}$ to $36^{\circ} \mathrm{S}$; therefore, a wide range of heading varieties are required for efficient and sustainable agriculture practices in these various geographic regions (Khush 1997). In rice, the flowering time or heading date is primarily controlled by the two florigens, Hd3a and RFT1 (Kojima et al. 2002; Komiya et al. 2008). Hd3a, an ortholog of 
Arabidopsis FT, interacts with 14-3-3 proteins in the apical cells of shoots, thereby yielding a complex that translocates to the nucleus and binds to OsFD1, which is a rice transcription factor homologue of Arabidopsis FD, to induce floral initiation (Taoka et al. 2011; Tsuji et al. 2013). RFT1, another ortholog of Arabidopsis FT, is a major floral activator under long-day (LD) conditions. The defective expression of RFT1 increases the heading date to more than 200 days, while, RFT1 overexpression reduces the heading date (Komiya et al. 2008; Komiya et al. 2009). During flower development, Hd3a and RFT1 translocate from the leaf to the apical meristem, thereby activating the expression of downstream genes, such as OsMADS14, OsMADS15 and OsMADS18, which regulate flower development (Tamaki et al. 2007; Komiya et al. 2008; Komiya et al. 2009; Kobayashi et al. 2012).

The regulation of $H d 3 a$ and RFT1 transcriptions involving multiple flowering pathways under SD and LD conditions have been reported (Greenup et al. 2009). In the OsGI-Hd1-Hd3a pathway, OsGI, similar to the Arabidopsis flowering time gene GIGANTA (GI), acts as a positive regulator upstream of $\mathrm{Hd} 1$ under both LD and SD conditions (Hayama et al. 2003). Hd1, which is orthologous to Arabidopsis CONSTANS (CO), regulates the expression of $\mathrm{Hd} 3 \mathrm{a}$. Hd1 stimulates the expression of $\mathrm{Hd} 3 \mathrm{a}$ under SD conditions but represses $\mathrm{Hd} 3 \mathrm{a}$ expression under LD conditions (Hayama and Coupland 2004). In the Ehd2-OsMADS50-Ehd1-RFT1 flowering pathway, Ehd1 represents another important expression regulator of $H d 3 a$ and RFT1. Ehd1 not only up-regulates the expression of $H d 3 a$ under SD conditions but also activates the expression of $H d 3 a$ and RFT1 under LD conditions (Doi et al. 2004; Komiya et al. 2009). Ehd1 expression is regulated by multiple floral regulators, such as OsMADS50, OsMADS51, OsMADS56 and Ehd2/ Osld1/RID1. Under SD conditions, the floral signal of OsGI is transmitted by OsMADS51, which is a MADSbox gene downstream of OsGI, to induce the expression of Ehd1 (Kim et al. 2007). Under LD conditions, Ehd1 is also up-regulated by OsMADS50 and Ehd2/Osld1/RID1, which is a Cys-2/His-2-type zinc finger transcription factor, while Ehd1 expression is repressed by OsMADS56 (Matsubara et al. 2008; Park et al. 2008; Wu et al. 2008; Ryu et al. 2009). Thus, the expression of $H d 3 a$ and RFT1 is regulated by multiple regulatory networks that govern flower initiation.

Rice MADS-box genes, which are required for floral organ differentiation and flowering time regulation, are grouped into the classes A, B, C, D and E based on the sequences homology and functional similarities to Arabidopsis homologues (Yamaguchi and Hirano 2006; Ciaffi et al. 2011). The MADS-box class A genes, OsMADS 14, 15 and 18, are downstream in the flowering signaling pathway and are regulated by $\mathrm{Hd} 3 \mathrm{a}$ and RFT1 under SD and LD conditions (Komiya et al. 2009; Kobayashi et al. 2012). Low expression levels of OsMADS14, OsMADS15 and OsMADS18 have been observed in the leaves at early developmental stages, while the expression of these genes increases when the plants reach the reproductive stage (Lee et al. 2004; Komiya et al. 2008; Kim et al. 2008). OsMADS14 and OsMADS15, which are homologous to Arabidopsis APETALA (AP1), which share a $72 \%$ amino acid sequence identity, interact with the E class protein, OsMADS1, during flower development regulation (Lim et al. 2000). OsMADS14 overexpression significantly reduces flowering time and causes flower bud development as early as transgenic rice cultured in regeneration medium (Jeon et al. 2000a). An OsMADS14 ortholog mutant, maintained vegetative phase (mvp), displayed no transition from vegetative to reproductive phase in einkorn wheat (Triticum monococcum) (Shitsukawa et al. 2007; Tsuji et al. 2008). Additionally, the overexpression of OsMADS18 revealed a short heading date in transgenic rice, while the heading date did not change in the knockout mutant (Fornara et al. 2004). For OsMADS15, a similar functional assay has not yet been reported. Therefore, the expression of OsMADS14 and OsMADS18 are closely associated with the reproductive phase transition. The expression analysis of these genes may elucidate the mechanism of the reproductive stage transition.

Rice OsMADS45 (also known as OsMADS7), which belongs to class $\mathrm{E}$, is specifically expressed in the stamen, pistil, and carpel, but not in the leaves and other vegetative tissues (Greco et al. 1997; Arora et al. 2007; Bai et al. 2008). E-functional genes are required for specifying organ identity of the inner three whorls of the floral organs in combination with A-, B-, C- and Dfunctional genes (Pelaz et al. 2000; Honma and Goto 2001). Many studies have indicated that OsMADS45 interacts with other MADS proteins to co-regulate the determination of floral organ identities (Seok et al. 2010). OsMADS13, which is a D class gene, interacts with OsMADS45 at the protein level to control ovule development during flower growth (Favaro et al. 2002; Dreni et al. 2007). OsMADS45 also associates with the B class proteins, OsMADS16 and OsMADS4, to form an OsMADS16-OsMADS4-OsMADS45 ternary complex and regulate the development of the lodicule and other floral organs (Moon et al. 1999a; Lopez-Dee et al. 1999; Seok et al. 2010). In addition to floral identity determination, the overexpression or deletion of $\mathrm{E}$ genes has been shown to alter the flowering time (Jeon et al. 2000a; Jeon et al. 2000b; Cui et al. 2010). For example, the OsMADS45 and OsMADS8 knockout plants displayed late flowering and a loss of floral identity (Cui et al. 2010), while the overexpression of OsMADS45 resulted in early 
flowering and affected other agricultural characteristics in transgenic rice (Jeon et al. 2000a). Similarly, a T-DNA activation-tagged mutant that has OsMADS45 activated and expressed in leaves and other tissues revealed early flowering and the same early flowering phenotype was observed by overexpressing OsMADS45 in a Taiwan rice variety TNG67. TNG67 is known as a photoperiod insensitive cultivar containing defective $H d 1$ and Ehd1 sequences as those of T65 (Doi et al. 2004), and malfunction of these two genes $H d 1$ and Ehd 1 delayed heading date up to 6.8 days and 17.4 days, respectively (Chen et al. 2010; Chien et al. 2011). However, the regulation mechanism causing early flowering in these OsMADS45 overexpression transgenic rice remains unknown. Thus, we expressed the OsMADS45 gene ectopically in TNG67 with the $h d 1$ and ehd1 genetic background to analyze the early flowering enhancing mechanism and to demonstrate the probable effects of OsMADS45 in regulating the flowering time when it was overexpressed. The overexpression of OsMADS45 not only induced the early expression of $H d 3 a$ and RFT1 but also reduced the expression of $H d 1$ at early developmental stages. Based on these data, we propose a model that explains the mechanism by which the ectopic expression of OsMADS45 causes early flowering in rice.

\section{Methods}

\section{Plant material and growth conditions}

The rice cultivar, Oryza sativa L. cv. TNG67, was analyzed in this study. The plants used for agronomic trait measurements were grown in an experimental field in Taichung, Taiwan (24 4' 40.2' N; $\left.120^{\circ} 43^{\prime} 0.61^{\prime \prime} \mathrm{E}\right)$. The plants used for the study of diurnal gene oscillations were grown in a growth chamber under a $14 \mathrm{~h}$ light/ $10 \mathrm{~h}$ dark cycle condition using fluorescent white light tubes (400 to $700 \mathrm{~nm}, 100 \mu \mathrm{mol} \mathrm{m} \mathrm{m}^{-2}$ ) at $28^{\circ} \mathrm{C}$.

\section{Measurement of photosynthesis rate}

The fully expanded upper three leaves of 60 DAI (days after imbibition) plants were used to measure the photosynthetic rate. The rate of photosynthetic $\mathrm{CO}_{2}$ assimilation was measured with a portable Plu-LCi ultra compact photosynthesis system (ADC BioScientific Ltd., Hertfordshire, UK). The measurements were performed from $9 \mathrm{AM}$ to $3 \mathrm{PM}$ with an external constant light source (Ess Capsule G4, Royal Philips Electronics, Amsterdam, Dutch). During the measurement procedure, the leaf temperature was $37^{\circ} \mathrm{C}$, the reference $\mathrm{CO}_{2}$ was $390 \mathrm{ppm}$, the relative air humidity was $60 \%$, and the flow rate to the leaf chamber was $300 \mathrm{~mL} \mathrm{~min}^{-1}$.

\section{Plasmid construction and rice transformation}

The full-length OsMADS45 cDNA was cloned by PCR amplification with the primer pair, BamOsMADS45, which containing aBam $\mathrm{HI}$ digesting site (Additional file 1:
Table S1) and introduced into a pGEM-T easy vector (Promega, http://www.promega.com/). The OsMADS45 cDNA was sub-cloned into a PLN vector containing the maize ubiquitin promoter (Ubi) and the nos terminator (Sun and Gubler 2004). The full-length construct (Ubi:OsMADS45) was fused with the pCAMBIA1301 vector, and the Agrobacterium tumefaciens strain, EHA105, was used for the rice transformations. Calli induced from seeds were co-cultured with A. tumefaciens, and the putative transgenic rice was regenerated from calli as described previously (Chen et al. 2002).

\section{Genomic DNA extraction and genes sequencing}

Total genomic DNA extraction was performed as described previously (Lin et al. 2009). The Hd1 and Ehd1 gene fragments were amplified by PCR with Promega Taq DNA polymerase. Two primer pairs (Hd1-1 and Hd1-2) were used to sequence the $H d 1$ gene. The primer pair Ehd1-1 was used to sequence the Ehd1 gene (Additional file 1: Table S1).

\section{Southern blot analysis}

Fifteen microgram of genomic DNA was digested with NcoI and separated on a $1 \%$ agarose gel. A GUS DNA fragment was amplified via PCR with the primer pair, GUS (Additional file 1: Table S1), and was used as probe. The GUS fragment was labeled with $\left(\alpha-\mathrm{P}^{32}\right) \mathrm{dCTP}$ using the Rediprime ${ }^{\mathrm{TM}}$ II DNA Labeling System (GE, http:// www.gelifesciences.com/). Southern blot analysis was performed as described previously (Ho et al. 2000).

\section{RNA isolation and semi-quantitative RT-PCR}

Total cellular RNA was extracted using TRIzol reagent (Invitrogen, http://www.invitrogen.com/), and the residual DNA was digested with RQ1 RNase-Free DNase (Promega) to minimize the contamination. For semiquantitative RT-PCR, first-strand cDNA was synthesized from $2 \mu \mathrm{g}$ of total RNA using M-MLV reverse transcriptase (Promega) in a $25 \mu \mathrm{L}$ reaction mixture containing $0.5 \mu \mathrm{g}$ of dT18 primer and 20 units of recombinant RNase inhibitor (TaKaRa, http://www.takara-bio.com/) according to the manufacturer's instructions. For the quantification of cDNA, rice Actin1 (Act1, Os03g0718100; Lin et al. 2009) was used to normalize the expression levels. The gene-specific primer pairs are listed in Additional file 1: Table S1. The PCR reactions involved $5 \mathrm{~min}$ of denaturation at $95^{\circ} \mathrm{C}$, followed by $95^{\circ} \mathrm{C}$ for $30 \mathrm{sec}, 62^{\circ} \mathrm{C}\left(54^{\circ} \mathrm{C}\right.$ for $\mathrm{Ehd} 1$ and $\mathrm{Hd} 3 \mathrm{a}$ ) for $30 \mathrm{sec}$ and $72^{\circ} \mathrm{C}$ for $45 \mathrm{sec}$. The PCR cycles used for each amplification are as follows: Act1, 28 cycles; Ehd1, 34 cycles; Ehd2, 34 cycles; Hd1, 34 cycles; Hd3a, 34 cycles; OsGI, 34 cycles; OsMADS7, 26 cycles; OsMADS14, 34 cycles; OsMADS18, 34 cycles; OsMADS50, 28 cycles and RFT1, 34 cycles. 


\section{Results}

Ectopic expression of OsMADS45 causes early flowering in the TNG67 rice variety

To analyze the early flowering enhancing mechanism of OsMADS45 and to demonstrate the probable effects of OsMADS45 in regulating the flowering time when it was overexpressed, we ectopically overexpressed OsMADS45 in the rice variety, TNG67, by transforming the Ubi: OsMADS45 construct containing the OsMADS45 gene driven by the maize ubiquitin promoter (Figure 1A) into the plant. After the transformation, more than 10 transgenic lines displayed early flowering and stunted growth, and this early flowering phenotype associated with OsMADS45 expression observed in the progenies of various transgenic lines was analyzed and verified for more than three generations. Four independent transgenic lines confirmed by Southern blot assay (Figure 1B) were selected for further investigation. RT-PCR analysis using RNA extracted from 20 DAI leaves revealed the overexpression of the OsMADS45 gene in all four Ubi: OsMADS45 transgenic lines (designated as 45OX1, $45 \mathrm{OX} 2,45 \mathrm{OX} 4$ and 45OX5), while no expression was detected in the wild-type (TNG67) and non-transgenic (NT) lines (Figure 1C). Although the RNA expression levels of OsMADS45 for these four transgenic lines were similar, the plant heights varied slightly (Figure 1D and $\mathrm{E}$ and Table 1).

The progenies of these four transgenic lines were grown in an isolated field for two crop seasons, and their agronomic traits, including heading date, plant height, panicle length, tiller number, fertility rate, grain size, number of spikelets per panicle, grain yield, shoot dry weight and photosynthetic rate, were measured and collected (Figure 1D and Table 1). The heading dates of the transgenic lines ranged from 46 to 51 DAI, which is approximately 40 days earlier than that of TNG67 plants (heading at $89 \mathrm{DAI}$ ) (Table 1). The difference in plant height between TNG67 and the transgenic lines was not significant until approximately $50 \mathrm{DAI}$, when the transgenic lines started heading (Figure 1D). The plant height of transgenic lines was retarded after 50 DAI, and the plants essentially stopped growing after 60 DAI (Figure 1D). The final plant height of the transgenic lines $(48-54 \mathrm{~cm})$ was approximately $40 \mathrm{~cm}$ shorter than
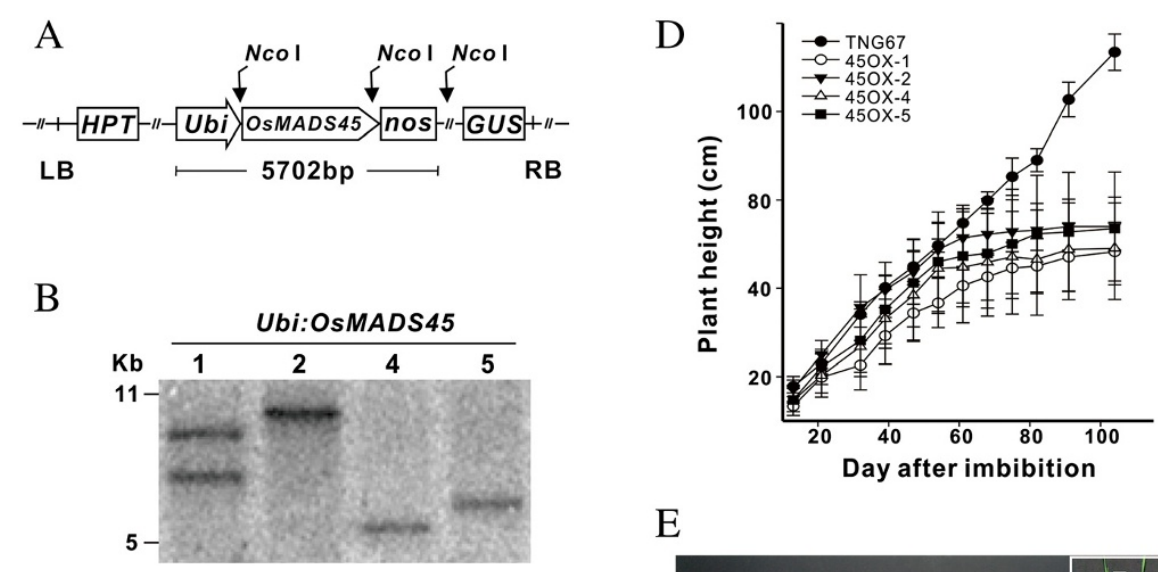

E
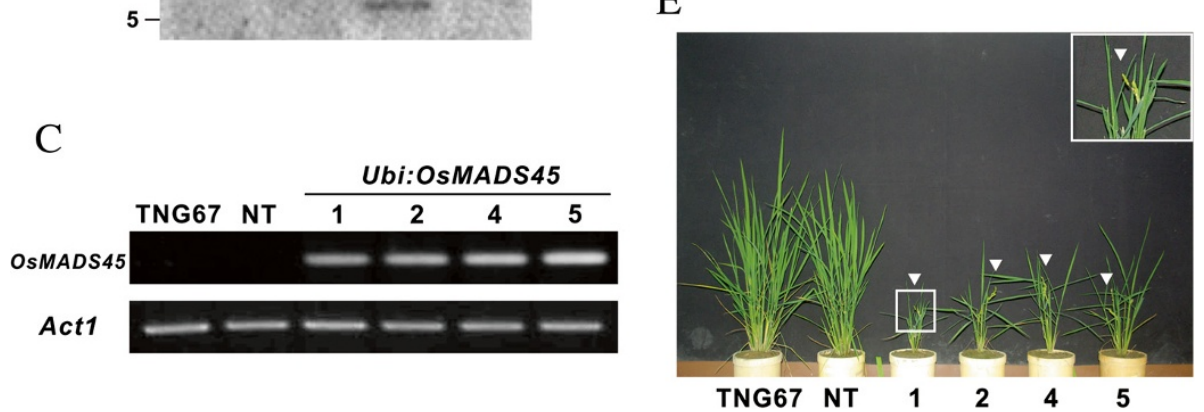

Figure 1 The transformation vector and the molecular and phenotype analysis of the Ubi:OsMADS45 transgenic rice. (A) A schematic diagram of the transformation vector, Ubi:OsMADS45. Full-length OsMADS45 cDNA driven by an ubiquitin (Ubi) promoter, and the nos terminator was inserted into the plasmid vector, pCAMBIA1301. (B) Southern blot analysis of four selected Ubi:OsMADS45 transgenic plants. Total genomic DNA isolated from four transgenic lines (450X1, 450X2, 450X4, and 450X5) was digested with Ncol and hybridized with a GUS DNA probe. (C) RT-PCR analysis of Ubi:OsMADS45 transgenic rice. Total RNA was isolated from 20 DAl leaves and analyzed using PCR primers specific for the OsMADS45 gene. Actin expression was assessed as an internal control. (D) The plant heights of TNG67 rice and the four transgenic lines from 20 to 100 DAl. The heights of 12-16 plants for each line were measured, and the error bars indicate the SE of the mean at each time point. (E) Morphological characteristics of TNG67 rice, non-transgenic (NT) and transgenic lines at 74 DAl. Arrowheads indicate the flowering panicles. Inset: an enlargement from the square portion indicated in line 1. DAl, days after imbibition; GUS, beta-glucuronidase; and HPT, hygromycin phosphor transferase. 
Table 1 Agronomic traits of TNG67 and Ubi:OsMADS45 transgenic rice plants

\begin{tabular}{|c|c|c|c|c|c|}
\hline Characteristics & TNG67 $^{\text {a }}$ & $450 X-1^{a}$ & $450 X-2^{a}$ & $450 X-4^{a}$ & $450 X-5^{a}$ \\
\hline Heading date $^{b}$ & $89 \pm 3$ & $51 \pm 2$ & $46 \pm 3$ & $48 \pm 4$ & $50 \pm 4$ \\
\hline Plant height ${ }^{c}(\mathrm{~cm})$ & $93.4 \pm 4.1$ & $48.3 \pm 6.4$ & $54.0 \pm 5.3$ & $49.1 \pm 11.6$ & $53.6 \pm 12.8$ \\
\hline Panicle length $(\mathrm{cm})$ & $20.2 \pm 2.3$ & $11.3 \pm 1.0$ & $12.7 \pm 2.2$ & $11.6 \pm 2.7$ & $11.5 \pm 2.0$ \\
\hline Tiller number $^{c}$ & $23 \pm 4$ & $13 \pm 3$ & $14 \pm 3$ & $15 \pm 4$ & $16 \pm 3$ \\
\hline Fertility rate ${ }^{d}(\%)$ & $95.8 \pm 1.8$ & $16.6 \pm 9.2$ & $45.2 \pm 15.9$ & $30.7 \pm 22.3$ & $37.6 \pm 20.1$ \\
\hline WTS $^{\text {e }}(\mathrm{g})$ & $22 \pm 0.8(100 \%)$ & $14.8 \pm 2.0(67 \%)$ & $16.2 \pm 1.3(73 \%)$ & $16.9 \pm 0.8(76 \%)$ & $14.6 \pm 0.6(66 \%)$ \\
\hline Number of spikelets per panicle & $105 \pm 15(100 \%)$ & $52 \pm 22(50 \%)$ & $52 \pm 12(50 \%)$ & $61 \pm 15(58 \%)$ & $53 \pm 17(50 \%)$ \\
\hline Grain yield ${ }^{f}(g)$ & $44.7 \pm 7.7(100 \%)$ & $3.5 \pm 2.0(8 \%)$ & $8.5 \pm 3.2(19 \%)$ & $7.5 \pm 6.7(17 \%)$ & $8.1 \pm 4.5(18 \%)$ \\
\hline Shoot dry weight ${ }^{g}(\mathrm{~g})$ & $46.9 \pm 8.6(100 \%)$ & $10.8 \pm 3.6(23 \%)$ & $17.7 \pm 4.4(38 \%)$ & $14.9 \pm 8.8(32 \%)$ & $16.3 \pm 8.3(35 \%)$ \\
\hline $\mathrm{PR}{ }^{\mathrm{h}}\left(\mu\right.$ mole $\left.\mathrm{m}^{-2} \mathrm{~s}^{-1}\right)$ & $10.5 \pm 1.1$ & $12.7 \pm 2.4$ & $13.2 \pm 2.2$ & $11.4 \pm 2.3$ & $10.4 \pm 2.4$ \\
\hline
\end{tabular}

${ }^{a}$ The plant number of TNG67 was 27, 450X-1 was 15, 450X-2 was 13, 450X-4 was 20, and 450X-5 was 20.

${ }^{b}$ Heading date was the date when the panicles protruding from the flag leaf sheath.

c Plant height and total tiller number were measured on 104 DAI.

${ }^{d}$ Fertility rate was the percentage of spikelets that set fertile seeds.

e Weight of one thousand seeds (WTS) was the weight counted with 50 seeds and multiplied by 20.

${ }^{f}$ Grain yield was the total weight of grains produced from one plant.

${ }^{g}$ Shoot dry weight was the weight of the up-ground part of rice without the grains.

${ }^{h}$ Photosynthetic rate (PR) was measured at noon on 68 DAI.

that of the TNG67 plants $(93 \mathrm{~cm})$. The transgenic plants measured approximately $50 \%$ of the TNG67 panicle length and reduced tiller number (13-15 vs. 23 tillers) and lower fertility rates $(17-45 \%$ vs. 96\%) (Table 1). Additionally, we found that the transgenic plants per grain weight measured $66-76 \%$ of that of the TNG67 plants, the number of spikelets per panicle for the transgenic plants was $50-58 \%$ of that observed with the TNG67 plants, and the grain yield for the transgenic plants was $8-19 \%$ of that observed with the TNG67 plants (Table 1). In terms of biomass production, the shoot dry weight of the transgenic plants was approximately $23-38 \%$ of that measured with the TNG67 plants; however, no difference in the photosynthetic rate was observed between the TNG67 plants and the transgenic lines.

\section{Ectopic expression of OsMADS45 does not alter the oscillation rhythm of flowering time regulators}

Flowering in many plants (including rice) is dependent on the day length. LD and SD photoperiod-sensitive rice initiate flowering dependent on a critical threshold associated with the proportion of diurnal hours. The light signals intercepted by photoreceptors and the length of photoperiod determined by the circadian clock regulators are integrated and lead to either the induction or suppression of flowering (Izawa, 2007). The floral regulators expressed in OsGI-Hd1-Hd3a and Ehd2-OsMADS50 -Ehd1-RFT1 flowering pathways are involved in the oscillation rhythm and controlled the flowering time (Ryu et al. 2009; Komiya et al. 2009). To study the oscillatory patterns of these floral regulators in 45OX-5 transgenic rice, leaf RNA samples were analyzed at each 4-hour interval under $14 \mathrm{~h}$ light/10 h dark cycle condition. The results showed that the diurnal oscillations of the floral regulators, including the light-induced $H d 3 a, R F T 1$ and Ehd1 regulators and the dark-induced $H d 1$ regulators, remained constant in 45OX transgenic lines (Figure 2). This result suggests that ectopic expression of OsMADS45 does not alter the diurnal oscillations of the examined floral regulators in 45OX transgenic rice on a TNG67 genetic background.

\section{Expression analysis of various floral regulatory genes in 450X transgenic rice}

In rice, two independent photoperiod pathways, involving the floral regulators, $\mathrm{Hd} 1$ and Ehd1, control flowering time via the regulation of Hd3a (Komiya et al. 2009). Hd1 expression is predominantly regulated by the circadian clock through OsGI signaling (Hayama et al. 2003), which regulates Ehd1 expression under SD conditions (Kim et al. 2007). Hd1, Ehd1 and Hd3a, are known to play a key role in photoperiod-controlled heading. Additionally, the OsMADS50-Ehd1-RFT1 pathway is involved in floral activation under LD conditions (Komiya et al. 2009). To understand the mechanism by which these floral regulators promote early flowering of the 45OX transgenic rice, we isolated RNA from the leaves at various growth stages, including $20,40,80$, and 120 DAI, and compared the expression levels with those of the wild-type TNG67. The results showed the expression patterns of the RFT1, Hd3a, Hd1, OsMADS14 and OsMADS18 genes in the $45 \mathrm{OX}$ transgenic rice varied from those observed with the TNG67 rice (Figure 3A), thereby suggesting that these genes may be involved in the process of early flowering in the $45 \mathrm{OX}$ transgenic 


\begin{tabular}{|c|c|}
\hline & TNG67 \\
\hline & $\begin{array}{llllllllll}2 & 6 & 10141822 & 2 & 6 & 1014 & 18 & 22 & b p\end{array}$ \\
\hline OsMADS45 & $-\infty----552(26)$ \\
\hline RFT1 & 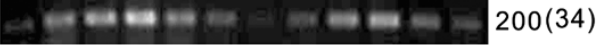 \\
\hline Ehd1 & $5-\infty-2$ \\
\hline OsMADS50 & 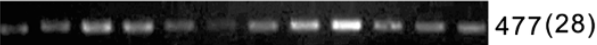 \\
\hline Ehd2 & 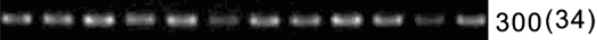 \\
\hline Hd3a & 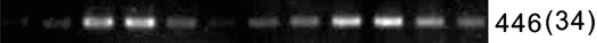 \\
\hline Hd1 & 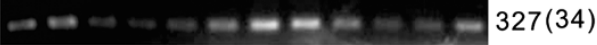 \\
\hline OsGI & 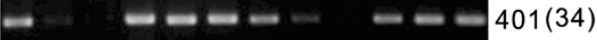 \\
\hline OsMADS14 & 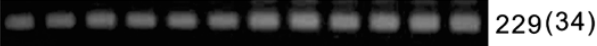 \\
\hline OsMADS18 & 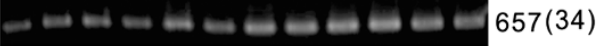 \\
\hline Act1 & $-2=-610(28)$ \\
\hline $\begin{array}{l}\text { Figure } 2 \text { The } \\
\text { regulatory ger } \\
\text { Hd1-Hd3a flov } \\
\text { TNG67 rice and } \\
14 \mathrm{~h} \text { light/10 h } \\
\text { collected and a } \\
\text { (Additional file } \\
\text { internal control } \\
\text { white bars indi }\end{array}$ & $\begin{array}{l}\text { diurnal expression patterns of the floral } \\
\text { nes of the Ehd2-OsMADS50-Ehd1-RFT1 and OsGI- } \\
\text { wering pathways. Leaf RNA samples from } 60 \text { DAI of } \\
\text { d Ubi:OsMADS45 transgenic rice (45OX) grown under } \\
\text { dark cycle condition at 4-hour intervals were } \\
\text { analyzed via RT-PCR using gene-specific primers } \\
\text { 1: Table S1). The actin gene was assessed as an } \\
\text { I. The black bars indicate the dark period and the } \\
\text { icate the light period. }\end{array}$ \\
\hline
\end{tabular}

rice. The expression levels of the floral regulatory genes observed for the two flowering pathways are detailed in the following sections.

The expression of RFT1 is activated at early developmental stages in 450X transgenic rice

The expression of the Ehd2, MADS50, Ehd1, and RFT1 genes of the Ehd2-OsMADS50-Ehd1-RFT1 flowering pathway were analyzed. RFT1 is a major floral activator under LD conditions, and its defective expression increases the heading date to above 200 days (Komiya et al. 2009), while, RFT1 overexpression reduces the heading date (Komiya et al. 2008). In the present study, the expression levels of RFT1 in 45OX transgenic rice were significantly higher than that observed in the TNG67 rice on 20 DAI. On 40 DAI, the RFT1 transcript levels observed in the TNG67 rice increased and reached the same levels of those observed in the 45OX transgenic rice. However, after 40 DAI, RFT1 expression levels decreased in the 45OX transgenic rice and increased in the TNG67 rice (Figure $3 \mathrm{~A}$ and $\mathrm{C}$ ).

Ehd1, a B-type response regulator, is an upstream positive regulator of RFT1 under LD conditions (Komiya et al. 2009), which showed no expression on 20 and 40 DAI and increased expression after 80 DAI (Figure 3A and D). Its expression pattern in $45 \mathrm{OX}$ transgenic rice was similar to that observed in the TNG67 plants
(Figure 3A and D). OsMADS50, a MICK-type MADSbox gene, is an epistatic active regulator of Ehd1 that was expressed throughout all development stages and displayed the same expression pattern in 45OX transgenic rice and the TNG67 plants (Figure 3A and E). Ehd2/Osld1/RID1, a zinc finger transcription factor orthologous with maize Indeterminate1 (ID1), is another upstream positive regulator of Ehd1 under LD conditions, which was expressed in all stages and failed to show different expression patterns between the $45 \mathrm{OX}$ transgenic rice and TNG67 rice (Figure 3A and F).

These results indicate that the overexpression of OsMADS45 activates the expression of RFT1 at early development stages, but fails to affect the expression of the upstream genes. As the heading of $45 \mathrm{OX}$ transgenic rice and TNG67 plants were detected soon after the high accumulation of the RFT1 transcript, we hypothesized that the activation and accumulation of RFT1 during the early development stages induces early flowering in 45OX transgenic rice.

The up-regulation of $\mathrm{Hd} 3 \mathrm{a}$ and down-regulation of $\mathrm{Hd} 1$ at early development stages were observed in 450X transgenic rice

The expression of the OsGI, Hd1 and $H d 3 a$ genes of the OsGI-Hd1-Hd3a flowering pathway were analyzed. $H d 3 a$, which is a rice florigen, has been shown to bind several proteins to induce flowering (Kojima et al. 2002) and was significantly expressed in the $45 \mathrm{OX}$ transgenic rice on 20 DAI, while the expression was decreased slightly on 40 DAI and then vanished after 80 DAI (Figure 3A and G). In contrast, $\mathrm{Hd} 3 \mathrm{a}$ expression was not detected in the TNG67 plants on 20 DAI, showed increased expression from 40 to $80 \mathrm{DAI}$, and then disappeared on $120 \mathrm{DAI}$ after flowering.

$H d 1$, another floral regulator orthologous with Arabidopsis $\mathrm{CO}$, is an upstream repressor of $\mathrm{Hd} 3 \mathrm{a}$ under LD conditions. Hd1 mRNA was approximately three-fold lower in the 45OX transgenic rice compared with the TNG67 rice on 20 DAI (Figure 3A and H). Nonetheless, no difference was observed on 40 DAI between the $45 \mathrm{OX}$ transgenic and TNG67 rice, the expression of Hd1 increased slightly on 80 DAI and then decreased in the $45 \mathrm{OX}$ transgenic rice, while decreased after $40 \mathrm{DAI}$ and on 80 DAI for in the TNG67 rice (Figure 3A and $\mathrm{H}$ ).

OsGI, a rice ortholog of Arabidopsis GI, is a positive regulator upstream of $H d 1$ that was expressed at all stages and failed to reveal different expression patterns between 45OX transgenic and TNG67 rice (Figure 3A and I). Our results indicate that OsMADS45 overexpression potentiates its effects on the OsGI-Hd1-Hd3a flowering signaling pathway downstream of OsGI and upstream of the $H d 1$ and $H d 3 a$ genes. The observations also reveal that the upregulation of $\mathrm{Hd} 3 \mathrm{a}$ at early development stages may play a 

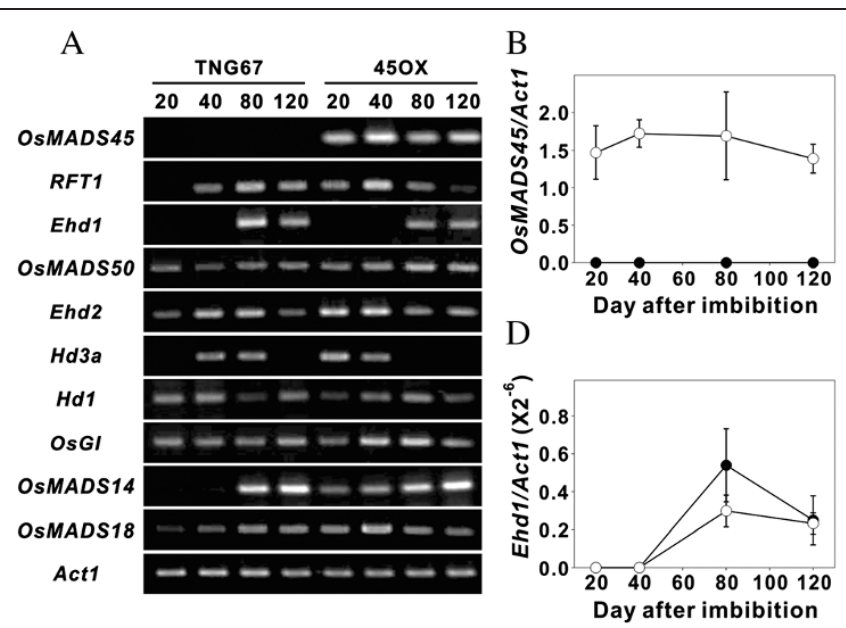

F

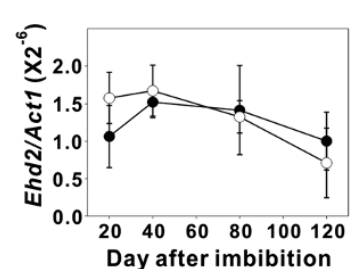

I

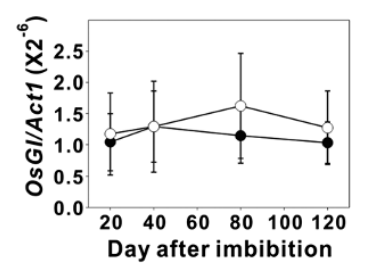

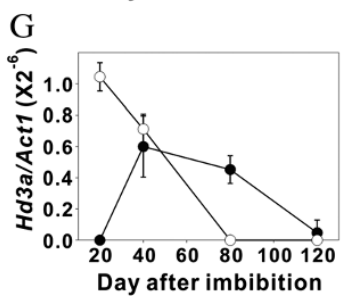

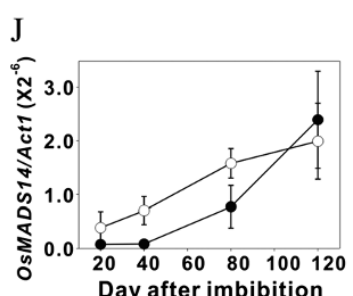

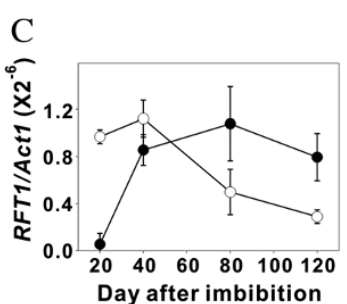

E
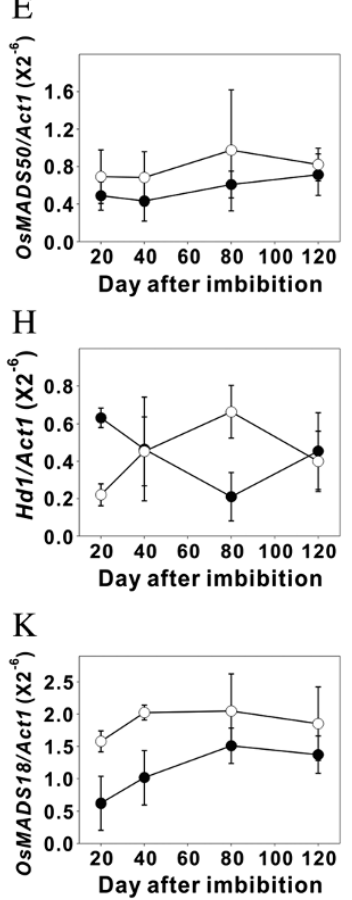

Figure 3 Expression analysis of the floral regulatory genes Ehd2, OsMADS50, Ehd1, RFT1, OsGl, Hd1, Hd3a, OsMADS14 and OsMADS18 in TNG67 and Ubi:OsMADS45 transgenic rice at various growth stages. (A) Representative RT-PCR analysis of the floral regulatory genes expression in TNG67 and Ubi:OsMADS45 transgenic plants (45OX) at various growth stages is shown. Leaf RNA was isolated from the plant samples of TNG67 and Ubi:OsMADS45 transgenic rice (45OX) approximately 2 to 4 hours after or before (for $\mathrm{Hd} 1$ gene analysis) dawn at 20, 40,80 and 120 DAl; the RNA was analyzed via RT-PCR using gene-specific primers (Additional file 1: Table S1). Act1 gene was used as an internal control. (B-K) Semiquantitative RT-PCR data of OsMADS45 (B), RFT1 (C), Ehd1 (D), OsMADS50 (E), Ehd2 (F), Hd3a (G), Hd1 (H), OsGl (I), OsMADS14 (J), and OsMADS18 (K) in TNG67 (black circle) and 450X (white circle) plants are shown. The $Y$ axes indicate the relative transcript levels of each gene. The transcript levels were quantified and normalized against actin RNA. The error bars indicate the SE for three replicate experiments.

role in the induction of early flowering in $45 \mathrm{OX}$ transgenic rice.

The expression of OsMADS14 and OsMADS18 is upregulated in 450X transgenic rice

To gain insight into the mechanism underlying the increase in RFT1 in 45OX transgenic rice, we assessed the gene expression levels of OsMADS14 and OsMADS18. OsMADS14 and OsMADS18, which are two Arabidopsis AP1-like MADS-box class A genes, are expressed in the leaves and inflorescences. Expressions of OsMADS14 and OsMADS18 was detectable during the vegetative stages and surged when the plants reach the reproductive stages (Lee et al. 2004; Komiya et al. 2008; Kim et al. 2008). These genes are recognized as the most downstream genes regulated by $H d 3 a$ and RFT1 under SD and LD conditions in both flowering-signaling pathways (Komiya et al. 2009). Although the apex-expressed but not the leaf-expressed OsMADS14 and OsMADS18 may directly participate in floral regulation, due to positive correlation between the leaf-expressed and apex- or floral-expressed OsMADS14/18 has been confirmed (Komiya et al. 2009; Sato et al. 2011), the leaf-expressed OsMADS14/18 were used in this study. Moreover, many previous studies used leaf-expressed OsMADS14/18 to analyze the reproductive phase transition and floral regulation as well (Lee et al. 2004; Doi et al. 2004; Kim et al. 2007; Komiya et al. 2008; Kim et al. 2008; Tanaka et al. 2011).

The expression of OsMADS14 was increased gradually from 20 DAI until 120 DAI in both the 45OX transgenic and TNG67 rice (Figure 3A and J). In the 45OX 
transgenic rice, similar expression levels were observed throughout all stages, while OsMADS18 expression increased slowly from 20 to 120 DAI in TNG67 (Figure 3A and $\mathrm{K})$. When comparing the expression levels of OsMADS14 and OsMADS18 in the 45OX transgenic and TNG 67 rice, we found that both genes were expressed at relatively higher levels in 45OX transgenic rice compared with the TNG67 rice throughout all stages with increasingly significant differences observed during the early stages (Figure $3 \mathrm{~A}, \mathrm{~J}$, and $\mathrm{K}$ ). As no OsMADS14 mRNA and relatively low levels of OsMADS18 were detected at the early development stages (20 to $40 \mathrm{DAI}$ ) in the wild-type TNG67 rice, the increased expression of OsMADS14 and OsMADS18 in $45 \mathrm{OX}$ transgenic rice may play a critical role in promoting the early transition from the vegetative stage to the reproductive stage, thereby resulting in early flowering.

\section{Genes Hd1 and Ehd1 are defective and an alternative spliced Hd1 mRNA was identified in TNG67 rice}

The $H d 1$ gene is highly variable in the cultivated rice Oryza sativa. The various alleles of $H d 1$ identified in the rice are believed to be selected by humans to adapt to the variable growing photoperiods (Izawa 2007; Takahashi and Shimamoto 2011). Hd1 and Ehd1 are key flowering time regulatory genes in rice; however, both genes have been identified to be non-functional in a Taiwan rice cultivar, Taichung 65 (T65), which shows little or no sensitivity to photoperiods (Doi et al. 2004). TNG67 is a hybrid cultivar descended from T65 plants and had decreased sensitivity to photoperiods. To verify $H d 1$ and Ehd1 gene integrity, both genes were sequenced in TNG67 rice. A total of 52 insertions or deletions and mismatched bases scattered between the $22^{\text {nd }}$ and $327^{\text {th }}$ bases near the $H d 1$ zinc finger domain in exon 1 and a 1912-bp insertion adjacent to the CCT domain in exon 2 were identified (Figure 4 and Additional file 2: Figure S1). The same substitution (a functional glycine replaced by a non- functional arginine) in the $219^{\text {th }}$ amino acid of Ehd1 found in T65 rice was also detected (Additional file 3: Figure S2). Although sequence variations in the $H d 1$ and Ehd1 genes were detected, the corresponding mRNAs were still regulated and expressed in TNG67 rice (Figures 2 and 3 ). We also found that the cDNA sequence of the $H d 1$ gene is 109 nucleotides shorter than that found in Nipponbare (Figure 4 and Additional file 2: Figure S1). Based on this cDNA sequence data obtained from the analysis of TNG67 rice, we concluded that this $H d 1$ transcript was generated by an alternative splice site (another AG inside the white box) located in exon 2 as indicated in Figure 4. These results suggest that the lack of function of $H d 1$ and Ehd1 in TNG67 rice (Chen et al. 2010; Chien et al. 2011) does not resulted from inhibited gene expressions but instead was caused either by alternative splicing and a DNA insertion into the $H d 1$ gene or by an amino acid substitution in the Ehd1 gene.

\section{Discussion}

The expression of the OsMADS45 gene is not only involved in the determinations of floral organ identity (Favaro et al. 2002; Dreni et al. 2007; Seok et al. 2010) but also involved in the regulation of flower initiation that affects the heading date of rice (Jeon et al. 2000a; Cui et al. 2010). The heading date of OsMADS45 RNAi knockout mutants was delayed by approximately 2 weeks (Cui et al. 2010). In contrast, the heading date of the OsMADS45 overexpressing transgenic rice instead of being delayed was reduced from the 50 days observed in the wild-type Nakdong rice to 41-48 days in a controlled growth room with $10 \mathrm{~h}$ of light per day (Jeon et al. 2000a). For further comparison, the heading date observed in our 45OX transgenic rice was approximately 40 days (50 vs. 90 days) earlier than that of the wild-type rice (TNG67) under field conditions of 11.5-13.5 h of light per day. The discrepancy in the shortening the

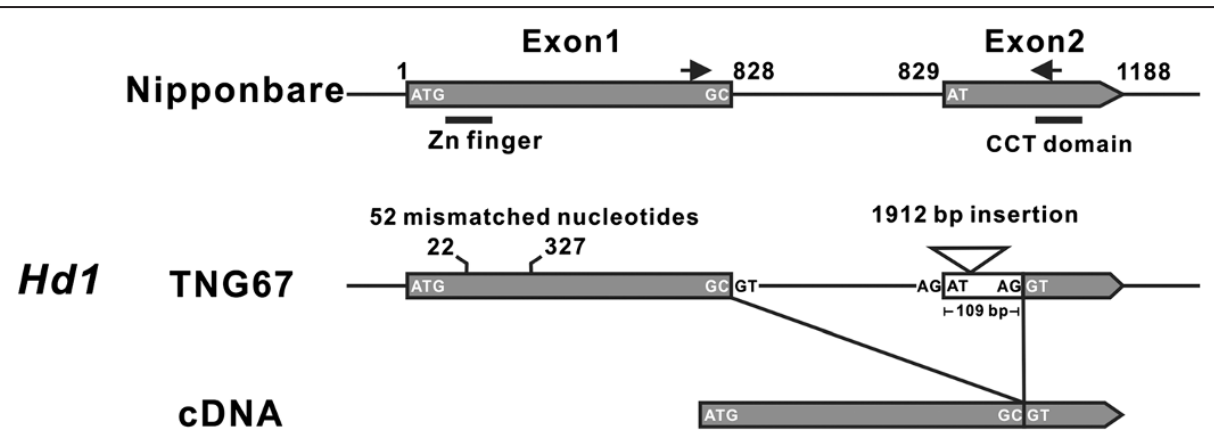

Figure 4 A schematic diagram showing the sequence variations and CDNA of Hd1 of TNG67 rice compare with Nipponbare plants. In TNG67 rice, a total of $52 \mathrm{in} /$ del and mismatched bases scattered between $22^{\text {nd }}$ and $327^{\text {th }}$ bases near the Hd1 zinc finger domain in exon 1 and a 1912 bp insertion adjacent to the CCT domain in exon 2 are shown (see detail in Additional file 2: Figure S1). The Hd1 cDNA of TNG67 with a potential alternative splice site located in exon 2 that causes the removal of a 109-bp of exon 2 and the 1912 bp insertion fragment is shown. 
heading date between the Jeon et al. study and our study may be due to the different rice cultivars, Nakdong and TNG67, with different Hd1 and Ehd1 genetic background (Figure 4, Additional file 2: Figure S1 and Additional file 3: Figure S2).

The Hd1 and Ehd1 genes are involved in photoperiodic regulation during floral initiation (Doi et al. 2004; Izawa 2007; Endo-Higashi and Izawa 2011). As in T65 (Doi et al. 2004) and TK8 (Lin et al. 2011) cultivars, both the $H d 1$ and Ehd1 genes are defective in TNG67 (Figure 4, Additional file 2: Figure S1 and Additional file 3: Figure S2). However, the RNA expressions of the defective $H d 1$ and Ehd1 genes can be detected in TNG67 and 45OX transgenic rice (Figure 3); this finding deviates from the results of the T65 study that showed no detection of the Hd1 RNA (Doi et al. 2004). While the transcript levels of $\mathrm{Hd} 1$ (a CO-like gene) was altered in the $45 \mathrm{OX}$ transgenic rice, the expression of the Ehd1 gene did not vary from that in the TNG67 (Figure 3A, D and $\mathrm{H}$ ). In the Nakdong rice, both the $H d 1$ and $E h d 1$ genes were functionally expressed (Kim et al. 2008) and displayed photoperiod sensitivity (Jeon et al. 2000a). Therefore, we hypothesize that the varying effects in the shortening of the heading dates between the 45OX transgenic TNG67 and Nakdong rice may be a result of the regulation and expression of the $H d 1$ gene. Our results showed that the $H d 1$ gene was downregulated during the juvenile stage in $45 \mathrm{OX}$ transgenic plants (Figure $3 \mathrm{H}$ ), and when this phenomenon was observed in the Nakdong rice, which expressed a functional $H d 1$ gene, would reduce the expression of $H d 3 a$ under SD conditions and abate the early flowering effect in transgenic Nakdong rice (Jeon et al. 2000a). However, additional analysis with our construct to transform Nakdong rice and grown in the same photoperiodic conditions would be required to address this discrepancy.

The floral time regulators in both the Ehd2OsMADS50-Ehd1-RFT1 LD activation pathway and the OsGI-Hd1-Hd3a LD suppression pathway exhibit diurnal oscillation patterns (Matsubara et al. 2008; Ryu et al. 2009). We assessed the daily expression levels of these genes at each 4-hour interval, and the results showed that the expression patterns of the floral regulatory genes, such as RFT1, Ehd1, OsMADS50, Ehd2, Hd3a, Hd1 and OsG1 did not vary between the 45OX transgenic rice and the host plant TNG67 (Figure 2), thereby indicating that the ectopic expression of OsMADS45 does not alter the diurnal oscillations of these genes. These observations suggest that the diurnal oscillations of these genes are not involved in reducing the flowering time in $45 \mathrm{OX}$ transgenic rice, and the normal diurnal oscillations of RFT1 and Hd3a observed in the hd1/ehd1 null cultivar, TNG67, imply that there are additional signal pathways other than those involving $H d 1$ and Ehd1 involved in the day/night rhythm of the RFT1 and Hd3a genes.

Although the diurnal oscillations of the examined floral time regulators were not altered, the RNA expression levels of RFT1 and $H d 3 a$, which are two rice orthologs of the Arabidopsis FT-like gene, were upregulated at a very early growth stages (20 DAI) in the $45 \mathrm{OX}$ transgenic rice (Figure 3 ). The up-regulation of the FT gene during different growth stages has been observed in transgenic Arabidopsis ectopically expressing MADS-box genes. For example, ectopic expression of orchid MADS1 in Arabidopsis enhances the expression of $F T$ and results in early flowering (Hsu et al. 2003), and the overexpression of AGAMOUS-LIKE6 (AGL6, also a MADS-box gene) causes the precocious flowering phenotype by enhancing the expression of $F T$ and the downstream gene AP1 (Koo et al. 2010). Similarly, the expression of RFT1 and $H d 3 a$ were up-regulated in the $45 \mathrm{OX}$ transgenic rice (Figure 3 ), thereby suggesting that the up-regulation of the RFT1 and $H d 3 a$ genes at a very early growth stage (20 DAI) may contribute to the early flowering phenomenon observed in the 45OX transgenic rice.

OsMADS14 and OsMADS18 are downstream of $\mathrm{Hd} 3 \mathrm{a}$ and RFT1 and regulate the identity of floral meristem development (Ciaffi et al. 2011; Kobayashi et al. 2012). In the floral tissue, OsMADS45 directly interacts with OsMADS6 and OsMADS18; OsMADS6 may further interact with OsMADS14 and OsMADS18 (Moon et al. 1999b) and OsMADS6 and OsMADS18 may form a ternary complex with the histone fold protein, OsNF-YB1 (Masiero et al. 2002). These interactions suggest that OsMADS45, OsMADS6, OsMADS14, and OsMADS18 co-regulate floral development. A number of studies indicated that the expression of the OsMADS14/15/18 were increased during the transition from the vegetative phase to the reproductive phase, thus, OsMADS14/15/18 have been considered as reproductive phase transition markers (Lee et al. 2004; Komiya et al. 2008; Kim et al. 2008; Gao et al. 2013). A recent study revealed that a null mutant of OsMADS6 (Osmads6-5) would down-regulate the expression of OsMADS7/45, but did not affect the expression of OsMADS14/15/18, therefore no flowering time change was observed (Duan et al. 2012). The same report showed over-expression of OsMADS6 resulted in over production of various flower organs, but has little influence on vegetative traits or the heading date (Duan et al. 2012). However, in the present study, over-expression of OsMADS45 resulted in an increased expression of OsMADS14 and OsMADS18 at early stages, approximately 20 to 40 days ahead of that of TNG67, and shortened the heading date and suppressed vegetative growth (Figure 3A, J and K). In contrast to $45 \mathrm{OX}$ transgenic rice that increased the 
expression of OsMADS14/18 and Hd3a/RFT1 and caused early flowering, a study using $M A D S 14 / 15 / 18 \mathrm{i}-$ pap2-1 quadruple knockdown mutant reduced the expression of $H d 3 a / R F T 1$ and resulted in delayed flowering (Kobayashi et al. 2012). Accordingly, they proposed that PAP2 and $A P 1$-like genes, such as $M A D S 14 / 15 / 18$, function upstream of $H d 3 a$ and RFT1 in leaves (Kobayashi et al. 2012). In summary, these observations suggested that 1) although OsMADS45 and OsMADS6 interact with OsMADS14 and OsMADS18 in regulating flower development, their effect on flowering time were different in overexpressing plants, 2) the downstream genes, such as OsMADS14 and OsMADS45 would regulate the expression of upstream genes $(H d 3 a / R F T 1)$ in either knockdown or over-expression transgenic rice, and 3) the early expression of the OsMADS14 and OsMADS18 in $45 \mathrm{OX}$ transgenic rice shortened the vegetative growth period which resulted in a flowering transition much earlier than that observed in TNG67 rice.

It is interesting to point out that the expression levels of RFT1 and $H d 3 a$ were dramatically decreased after heading at $50 \mathrm{DAI}$ in $45 \mathrm{OX}$ transgenic rice or after heading at 90 DAI in TNG67 (Table 1; Figure 3C, G), and the expression of RFT1 and $H d 3 a$ were not well correlated with the constitutively expression of OsMADS45 in $45 \mathrm{OX}$ transgenic rice after heading (Figure 3B, C, G). The increased expression of RFT1 and $H d 3 a$ at a higher level before heading and then at a significantly reduced level after heading were observed (Matsubara et al. 2008; Komiya et al. 2008; Kim et al. 2008; Ryu et al. 2009). These observations suggested that both the expression of RFT1 and $H d 3 a$ were developmentally regulated by factors that could override the overexpression effect of OsMADS45 after heading in $45 \mathrm{OX}$ transgenic rice. Although OsMADS14 and OsMADS18 are downstream of $H d 3 a$ and RFT1, the expression of OsMADS14 and OsMADS18 were not positively correlated with the expression of $H d 3 a$ and RFT1 after heading (Kim et al. 2008). While the expression levels of OsMADS14 and OsMADS18 increased throughout the entire growth season, the expression of $H d 3 a$ and RFT1 was reduced after heading and this pattern was also observed in TNG67 and in 45OX transgenic rice. This observation suggested that the increased expression of OsMADS14 and OsMADS18 after heading was controlled by factors in addition to $H d 3 a$ and RFT1. Based on the understanding of these flower development regulation pathways, we hypothesize that the early increased expression of the OsMADS14 and OsMADS18 genes in the 45OX transgenic rice may be caused by either the up-regulation of $H d 3 a$ and RFT1 or directly induced by the constitutive expression of OsMADS45 (Figure 5).

In the present study, we over-expressed the OsMADS45 gene in TNG67 rice and showed that not only was the heading date reduced but also revealed that many other characteristics, such as plant height, shoot dry weight and the number of spikelets per panicle, were dramatically suppressed (Figure $1 \mathrm{E}$ and Table 1). A previous study, which involved the introducing of functional Hd1 and/or Ehd1 genes into T65 rice under different photoperiodic conditions, revealed that the combination of $H d 1$ and Ehd 1 expression reduced the number of primary branches in a panicle, thereby resulting in smaller spiketlet numbers per panicle independent of the control
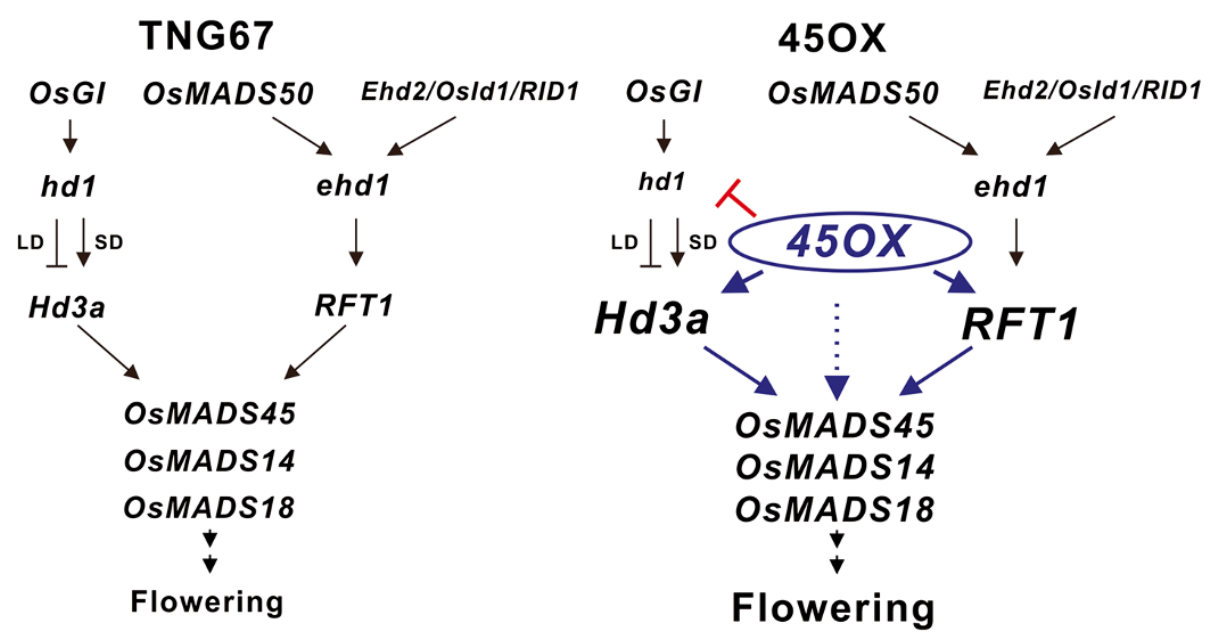

Figure $5 \mathrm{~A}$ model of the early flowering signal pathway in 450X transgenic rice. The ectopic expression of OsMADS45 activates the upstream genes Hd3a and RFT1 at early developmental stages and upregulates the expression of OsMADS14 and OsMADS18 either via the upregulated of Hd3a and RFT1 or direct induction via the constitutive expression of OsMADS45. The expression of OsMADS45 represses the expression of $\mathrm{Hd} 1$ at early developmental stages. The increased expression of the OSMADS14 and OSMADS18 genes caused early flowering in 450X transgenic rice. However, the ectopic expression of OsMADS45 does not alter the expression of OsMADS50, Ehd1, Ehd2/Os/d1/RID1 and OsGl. 
of flowering time (Endo-Higashi and Izawa 2011). However, as observed in the T65 rice, TNG67 rice are defective in both the Hd1 and Ehd 1 genes (Figure 4 and Additional file 3: Figure S2); therefore, the reduction in spikelet numbers per panicle and other characteristics observed in our 45OX transgenic rice could not have been affected by the function of $H d 1$ and Ehd1. Additionally, no reduction in the photosynthetic rate was observed in $45 \mathrm{OX}$ transgenic rice (Table 1); this finding suggests that overexpression of OsMADS45 does not affect the photosynthetic system and the reduction in shoot dry weight in $45 \mathrm{OX}$ transgenic rice was not caused by a reduction in photosynthesis. Furthermore, plant height and grain productivity associated with the flowering time regulators, Ghd7 and Ghd8, have been previously reported (Xue et al. 2008; Yan et al. 2010). The report has shown that the enhanced expression of Ghd7 under LD conditions could delay heading and increase both plant height and panicle size (Xue et al. 2008). Similarly, Ghd8 plays a pleiotropic role in regulating grain productivity and heading date depending on its genetic background, which reveals a positive correlation between flowering time and grain productivity (Yan et al. 2010). Accordingly, we propose that many of the agronomic traits, such as shorter plant height, shorter panicle length, less tiller, less shoot dry weight and less grain yield in this $450 \mathrm{OX}$ transgenic rice might be the result of the effects of early flowering that reduced the vegetative growth periods from 89 days in TNG67 rice to 50 days.

\section{Conclusions}

Based on our results, we propose a model to explain the signaling cascade in the early flowering phenotype of $45 \mathrm{OX}$ transgenic rice (Figure 5). The ectopic expression of OsMADS45 activates the upstream genes, RFT1 and $H d 3 a$, at early development stages and up-regulates the expression of OsMADS14 and OsMADS18 via either the up-regulation of $H d 3 a$ and RFT1 or direct induction via the constitutive expression of OsMADS45, thereby promoting early flowering. Additionally, the regulation and function of the various $H d 1$ alleles may be involved in the different shortened effects on the heading dates observed between the 45OX transgenic TNG67 rice and Nakdong rice under the SD condition. In summary, our study illustrates the overexpression function of OsMADS45 in initiating a possible bottom-up activation of the MADS-box genes signaling pathway that enhances early flower initiation, and the study using TNG67 rice may also represent another useful approach to study the flowering mechanism of other floral regulators to bypass the complex photoperiodic responses that are regulated by the $H d 1$ and Ehd1 signaling pathways.

\section{Additional files}

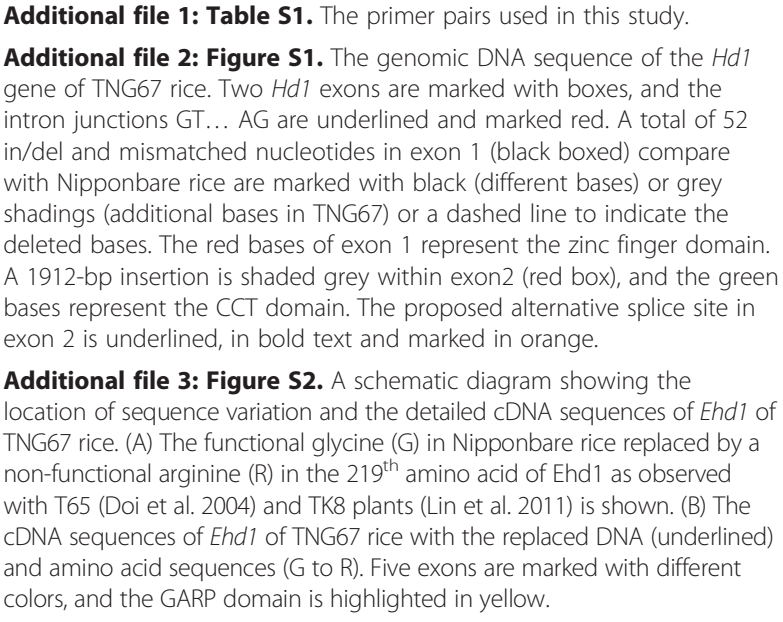

Competing interests

The authors declare that they have no competing interests.

\section{Authors' contributions}

JDW carried out the laboratory studies and made interpretation of data and drafted the manuscript. SFL carried out the mutant selections and identified the genes. YSL identified the function of genes. PJC generated the transgenic plants and carried out the initial assays. SYL carried out the field works. TYH drafted and revised the manuscript. JHL designed the experiments and supply facilities. LJC designed the experiments, drafted and finalized the manuscript. All authors read and approved the final manuscript.

\section{Acknowledgements}

We thank Show-Ya Yang, I-Wen Wang and Wen-Bin Tseng for their technical assistance with the transgenic rice plants, thank Dr. Yann-Rong Lin for the critical suggesting in revising this manuscript. This work was supported by grants from the National Science Council (NSC-97-2317-B-005-002 and NSC98-2628-B-005-018 to L-J.C.) of Taiwan and in part by the Ministry of

Education, Taiwan under the ATU plan.

\section{Author details}

${ }^{1}$ Department of Life Sciences, National Chung Hsing University, Taichung 402, Taiwan. ${ }^{2}$ Institute of Molecular Biology, National Chung Hsing University, Taichung 402, Taiwan. ${ }^{3}$ Agricultural Biotechnology Center, National Chung Hsing University, Taichung 402, Taiwan. ${ }^{4}$ Present address: Institute of Molecular Biology, Academia Sinica, Taipei 115, Taiwan. ${ }^{5}$ Present address: Institute of Plant and Microbiology, Academia Sinica, Taipei 115, Taiwan.

Received: 11 October 2012 Accepted: 20 March 2013

Published: 21 August 2013

\section{References}

Arora R, Agarwal P, Ray S, Singh AK, Singh VP, Tyagi AK, Kapoor S (2007) MADSbox gene family in rice: genome-wide identification, organization and expression profiling during reproductive development and stress. BMC Genomics 8:242

Bai X, Wang Q, Chu C (2008) Excision of a selective marker in transgenic rice using a novel Cre/loxP system controlled by a floral specific promoter Transgenic Res 17:1035-1043

Chen PW, Lu CA, Yu TS, Tseng TH, Wang CS, Yu SM (2002) Rice a-amylase transcriptional enhancers direct multiple mode regulation of promoters in transgenic rice. J Biol Chem 277:13641-13649

Chen CS, Chen RK, Chin HH, Lin YR (2010) Introgression of three heading date gene, hd1, Hd6, and ehd1, to Oryza sativa L. japonica cv. Koshihikari by marker-assisted selection. Crop, Environ. \& Bioinformatics 7:1-20

Chien HT, Chen RK, Hour AL, Chen CS, Lin YR (2011) The effects of Hd1, Hd6, and Ehd1 on rice heading date. Crop, Environ. \& Bioinformatics 8:45-57 
Ciaffi M, Paolacci AR, Tanzarella OA, Porceddu E (2011) Molecular aspects of flower development in grasses. Sex Plant Reprod 24:247-282

Cui RF, Han JK, Zhao SH, Su KM, Wu F, Du XQ, Xu QJ, Chong K, Theißen G, Meng $Z$ (2010) Functional conservation and diversification of class $E$ floral homeotic genes in rice (Oryza sativa). Plant J 61:767-781

Doi K, Izawa T, Fuse T, Yamanouchi U, Kubo T, Shimatani Z, Yano M, Yoshimura A (2004) Ehd1, a B-type response regulator in rice, confers short-day promotion of flowering and controls FT-like gene expression independently of $\mathrm{Hd} 1$. Genes Dev 18:926-936

Dreni L, Jacchia S, Fornara F, Fornari M, Ouwerkerk PBF, An G, Colombo L, Kater MM (2007) The D-lineage MADS-box gene OsMADS13 controls ovule identity in rice. Plant J 52:690-699

Duan Y, Xing Z, Diao Z, Xu W, Li S, Du X, Wu G, Wang C, Lan T, Meng Z, Liu H, Wang F, Wu W, Xue Y (2012) Characterization of Osmads6-5, a null allele, reveals that OsMADS6 is a critical regulator for early flower development in rice (Oryza sativa L.). Plant Mol Biol 80:429-442

Endo-Higashi N, Izawa T (2011) Flowering time genes Heading date 1 and Early heading date 1 together control panicle development in rice. Plant Cell Physiol 52:1083-1094

Favaro R, Immink RGH, Ferioli V, Bernasconi B, Byzova M, Angenent GC, Kater M, Colombo L (2002) Ovule-specific MADS-box proteins have conserved protein-protein interactions in monocot and dicot plants. Mol Genet Genomics 268:152-159

Fornara F, Pařenicova' L, Falasca G, Pelucchi N, Masiero S, Ciannamea S, LopezDee Z, Altamura MM, Colombo L, Kater MM (2004) Functional Characterization of OSMADS18, a Member of the AP1/SQUA Subfamily of MADS-box Genes. Plant Physiol 135:2207-2219

Gao H, Zheng XM, Fei G, Chen J, Jin M, Ren Y, Wu W, Zhou K, Sheng P, Zhou F, Jiang L, Wang J, Zhang X, Guo X, Wang JL, Cheng Z, Wu C, Wang H, Wan JM (2013) Ehd4 encodes a novel and oryza-genus-specific regulator of photoperiodic flowering in rice. PLoS Genet 9:e1003281

Greco R, Stagi L, Colombo L, Angenent GC, Sari-Gorla M, Pè ME (1997) MADS-box genes expressed in developing inflorescences of rice and sorghum. Mol Gen Genet 253:615-623

Greenup A, Peacock WJ, Dennis ES, Trevaskis B (2009) The molecular biology of seasonal flowering-responses in Arabidopsis and the cereals. Ann Bot 103:1165-1172

Hayama R, Coupland G (2004) The molecular basis of diversity in thephotoperiodic flowering responses of Arabidopsis and rice. Plant Physiol 135:677-684

Hayama R, Yokoi S, Tamaki S, Yano M, Shimamoto K (2003) Adaptation of photoperiodic control pathways produces short-day flowering in rice. Nature 422:719-722

Ho SL, Tong WF, Yu SM (2000) Multiple mode regulation of a cysteine proteinase gene expression in rice. Plant Physiol 122:57-66

Honma T, Goto K (2001) Complexes of MADS-box proteins are sufficient to convert leaves into floral organs. Nature 409:525-529

Hsu HF, Huang CH, Chou LT, Yang CH (2003) Ectopic expression of an orchid (Oncidium Gower Ramsey) AGL6-like gene promotes flowering by activating flowering time genes in Arabidopsis thaliana. Plant Cell Physiol 44:783-794

Izawa T (2007) Adaptation of flowering-time by natural and artificial selection in Arabidopsis and rice. J Exp Bot 58:3091-3097

Jeon JS, Lee S, Jung KH, Yang WS, Yi GH, Oh BG, An G (2000a) Production of transgenic rice plants showing reduced heading date and plant height by ectopic expression of rice MADS-box genes. Mol Breed 6:581-592

Jeon JS, Jang S, Lee S, Nam J, Kim C, Lee SH, Chung YY, Kim SR, Lee YH, Cho YG, An $\mathrm{G}$ (2000b) leafy hull sterile1 is a homeotic mutation in a rice MADS-box gene affecting rice flower development. Plant Cell 12:871-884

Khush GS (1997) Origin, dispersal, cultivation and variation of rice. Plant Mol Biol 35:25-34

Kim SL, Lee S, Kim HJ, Nam HG, An G (2007) OsMADS51 is a short-day flowering promoter that functions upstream of Ehd1, OsMADS14, and Hd3a. Plant Physiol 145:1484-1494

Kim SK, Yun CH, Lee JH, Jang YH, Park HY, Kim JK (2008) OsCO3, a CONSTANS-LIKE gene, controls flowering by negatively regulating the expression of $F T$-like genes under SD conditions in rice. Planta 228:355-365

Kobayashi K, Yasuno N, Sato Y, Yoda M, Yamazaki R, Kimizu M, Yoshida H, Nagamura Y, Kyozuka J (2012) Inflorescence meristem identity in rice is specified by overlapping functions of three AP1/FUL-Like MADS-box genes and PAP2, a SEPALLATA MADS-box gene. Plant Cell 24:1848-1859

Kojima S, Takahashi Y, Kobayashi Y, Monna L, Sasaki T, Araki T, Yano M (2002) $\mathrm{Hd} 3 \mathrm{a}$, a rice ortholog of the Arabidopsis FT Gene, promotes transition to flowering downstream of Hd1 under short-day conditions. Plant Cell Physiol 43:1096-1105

Komiya R, Ikegami A, Tamaki S, Yokoi S, Shimamoto K (2008) Hd3a and RFT1 are essential for flowering in rice. Development 135:767-774

Komiya R, Yokoi S, Shimamoto K (2009) A gene network for long-day flowering activates RFT1 encoding a mobile flowering signal in rice. Development 136:3443-3450

Koo SC, Bracko O, Park MS, Schwab R, Chun HJ, Park KM, Seo JS, Grbic V Balasubramanian S, Schmid M, Godard F, Yun DJ, Lee SY, Cho MJ, Weigel D, Kim MC (2010) Control of lateral organ development and flowering time by the Arabidopsis thaliana MADS-box gene AGAMOUS-LIKE6. Plant J 62:807-816 Koornneef M, Alonso-Blanco C, Peeters AJM, Soppe W (1998) Genetic control of flowering time in Arabidopsis. Annu Rev Plant Physiol Plant Mol Biol 49:345-370

Lee S, Kim J, Han JJ, Han MJ, An G (2004) Functional analyses of the flowering time gene OSMADS50, the putative SUPPRESSOR OF OVEREXPRESSION OF CO 1/AGAMOUS-LIKE 20 (SOC1/AGL20) ortholog in rice. Plant J 38:754-764

Lim J, Moon YH, An G, Jang SK (2000) Two rice MADS domain proteins interact with OsMADS1. Plant Mol Biol 44:513-527

Lin SY, Wang JD, Lin JH (2009) Expression of Trigonopsis variabilis D-amino acid oxidase in transgenic rice for cephalosporin production. Bot Stud 50:181-192

Lin YR, Wu SC, Chen SE, Tseng TH, Chen CS, Kuo SC, Wu HP, Hsing YIC (2011) Mapping of quantitative trait loci for plant height and heading date in two inter-subspecific crosses of rice and comparison across Oryza genus. Bot Stud 52:1-14

Lopez-Dee ZP, Wittich P, Enrico Pè M, Rigola D, Del Buono I, Gorla MS, Kater MM, Colombo L (1999) OsMADS13, a novel rice MADS-box gene expressed during ovule development. Dev Genet 25:237-244

Masiero S, Imbriano C, Ravasio F, Favaro R, Pelucchi N, Gorla MS, Mantovani R, Colombo L, Kater MM (2002) Ternary complex formation between MADS-box transcription factors and the histone fold protein NF-YB. J Biol Chem 277:26429-26435

Matsubara K, Yamanouchi U, Wang ZX, Minobe Y, Izawa T, Yano M (2008) Ehd2, a rice ortholog of the maize INDETERMINATE1 Gene, promotes flowering by upregulating Ehd1. Plant Physiol 148:1425-1435

Moon YH, Jung JY, Kang HG, An G (1999a) Identification of a rice APETALA3 homologue by yeast two-hybrid screening. Plant Mol Biol 40:167-177

Moon YH, Kang HG, Jung JY, Jeon JS, Sung SK, An G (1999b) Determination of the motif responsible for interaction between the rice APETALA1 AGAMOUS-LIKE9 family proteins using a yeast Two-hybrid system. Plant Physiol 120:1193-1203

Park SJ, Kim SL, Lee S, Je BI, Piao HL, Park SH, Kim CM, Ryu CH, Park SH, Xuan YH, Colasanti J, An G, Han CD (2008) Rice Indeterminate 1 (Os/d1) is necessary for the expression of Ehd1 (Early heading date 1) regardless of photoperiod. Plant J 56:1018-1029

Pelaz S, Ditta GS, Baumann E, Wisman E, Yanofsky MF (2000) B and C floral organ identity functions require SEPALLATA MADS-box genes. Nature 405:200-203

Ryu CH, Lee S, Cho LH, Kim SL, Lee YS, Choi SC, Jeong HJ, Yi J, Park SJ, Han CD, An G (2009) OsMADS50 and OsMADS56 function antagonistically in regulating long day (LD)-dependent flowering in rice. Plant Cell Environ 32:1412-1427

Sato $Y$, Antonio BA, Namiki N, Takehisa H, Minami H, Kamatsuki K, Sugimoto K, Shimizu Y, Hirochika H, Nagamura Y (2011) RiceXPro: a platform for monitoring gene expression in japonica rice grown under natural field conditions. Nucleic Acids Res 39:D1141-D1148

Seok HY, Park HY, Park Jl, Lee YM, Lee SY, An G, Moon YH (2010) Rice ternary MADS protein complexes containing class B MADS heterodimer. Biochem Biophys Res Commun 401:598-604

Shitsukawa N, Ikari C, Shimada S, Kitagawa S, Sakamoto K, Saito H, Ryuto H, Fukunishi N, Abe T, Takumi S, Nasuda S, Murai K (2007) The einkorn wheat (Triticum monococcum) mutant, maintained vegetative phase, is caused by a deletion in the VRN1 gene. Genes Genet Syst 82:167-170

Sun TP, Gubler F (2004) Molecular mechanism of gibberellin signaling in plants. Annu Rev Plant Biol 55:197-223

Takahashi Y, Shimamoto K (2011) Heading date 1 (Hd1), an ortholog of Arabidopsis CONSTANS, is a possible target of human selection during domestication to diversify flowering times of cultivated rice. Genes Genet Syst 86:175-182

Tamaki S, Matsuo S, Wong HL, Yokoi S, Shimamoto K (2007) Hd3a protein is a mobile flowering signal in rice. Science 316:1033-1036

Tanaka N, Itoh H, Sentoku N, Kojima M, Sakakibara H, Izawa T, Itoh Jl, Nagato Y (2011) The COP1 ortholog PPS regulates the juvenile-adult and vegetativereproductive phase changes in rice. Plant Cell 23:2143-2154 
Taoka Kl, Ohki I, Tsuji H, Furuita K, Hayashi K, Yanase T, Yamaguchi M, Nakashima C, Purwestri YA, Tamaki S, Ogaki Y, Shimada C, Nakagawa A, Kojima C, Shimamoto K (2011) 14-3-3 proteins act as intracellular receptors for rice Hd3a florigen. Nature 476:332-335

Tsuji H, Tamaki S, Komiya R, Shimamoto K (2008) Florigen and the photoperiodic control of flowering in rice. Rice 1:25-35

Tsuji H, Nakamura H, Taoka K, Shimamoto K (2013) Functional diversification of FD transcription factors in rice, components of florigen activation complexes. Plant Cell Physiol 54:385-397

Wilczek AM, Burghardt LT, Cobb AR, Cooper MD, Welch SM, Schmitt J (2010) Genetic and physiological bases for phenological responses to current and predicted climates. Philos Trans R Soc Lond B Biol Sci 365:3129-3147

Wu C, You C, Li C, Long T, Chen G, Byrne ME, Zhang Q (2008) RID1, encoding a Cys2/His2-type zinc finger transcription factor, acts as a master switch from vegetative to floral development in rice. Proc Natl Acad Sci U S A 105:12915-12920

Xue W, Xing Y, Weng X, Zhao Y, Tang W, Wang L, Zhou H, Yu S, Xu C, Li X Zhang Q (2008) Natural variation in Ghd7 is an important regulator of heading date and yield potential in rice. Nat Genet 40:761-767

Yamaguchi T, Hirano HY (2006) Function and diversification of MADS-box genes in rice. ScientificWorldJournal 6:1923-1932

Yan WH, Wang P, Chen HX, Zhou HJ, Li QP, Wang CR, Ding ZH, Zhang YS, Yu SB, Xing YZ, Zhang QF (2010) A major QTL, Ghd8, plays pleiotropic roles in regulating grain productivity, plant height, and heading date in rice. $\mathrm{Mol}$ Plant 4:319-330

doi:10.1186/1999-3110-54-12

Cite this article as: Wang et al:: Ectopic expression of OsMADS45

activates the upstream genes $H d 3 a$ and RFT1 at an early development stage causing early flowering in rice. Botanical Studies 2013 54:12.

\section{Submit your manuscript to a SpringerOpen ${ }^{\circ}$ journal and benefit from:}

- Convenient online submission

- Rigorous peer review

- Immediate publication on acceptance

- Open access: articles freely available online

- High visibility within the field

- Retaining the copyright to your article

Submit your next manuscript at $>$ springeropen.com 